\title{
A microreactor-based system for the study of fast exothermic reactions in liquid phase: characterization of the system
}

\author{
M.-A. Schneider ${ }^{1}$, T. Maeder ${ }^{2}$, P. Ryser ${ }^{2}$, F. Stoessel ${ }^{1}$ \\ 1) Groupe de Sécurité des Procédés Chimiques (GSPC), Faculté des Sciences de Base, Institut de Sciences des Procédés (ISP), \\ EPFL, Lausanne, Switzerland \\ 2) Laboratoire de Production Microtechnique (LPM), Faculté des Sciences de l'ingénieur, Institut de Production et Robotique \\ (IPR), EPFL, Lausanne, Switzerland
}

Version of record: Chemical Engineering Journal 101 (1-3), 241-250, 2004.

http://hdl.handle.net/10.1016/j.cej.2003.11.005

\begin{abstract}
A new system combining a microreactor and a microcalorimetric chip was investigated. The small size of the microreactor channel permits maintaining isothermal conditions necessary for the kinetic characterization of highly exothermic reactions. These conditions are not easily obtained in classical calorimetric systems.

The degree of mixing in the microchannel, which plays an important role for the characterization of fast reactions, was determined experimentally using an iodate - iodide system and confirmed by calculations. The calorimetric system was calibrated by performing the neutralization reaction of $\mathrm{NaOH}$ with $\mathrm{H}_{2} \mathrm{SO}_{4}$ in the microchannel. Several optimizations of the microreactor design allowed to raise the sensitivity of the system and to preheat the entering fluids. The results obtained were repeatable and reproducible.

The establishment of the heat balance and the evaluation of the various resistances allowed a better global understanding of the system.
\end{abstract}

Keywords: Microreactor; Calorimetric system; Microchannel; Exothermic reactions

\section{Introduction}

Fast exothermic reactions raise several problems. The control of the reactor temperature and a sufficiently rapid mixing are difficult to achieve even at laboratory scale. This may result into selectivity and also safety problems.

The isothermal conditions necessary for the determination of reaction kinetics are almost impossible to achieve even in calorimetric systems. Moreover, as the time constant of the system is longer than the time constant of the reaction, the precision of the results is low and mathematical tools are needed to deconvolute the curves.

The use of microreactors may present several advantages in such cases [1]. The high surface-to-volume ratio improves the response time, leads to efficient heat transfer and allows maintaining isothermal conditions. Moreover, microreactors can be designed to provide rapid mixing [2].

In this work, a new calorimetric system using a continuous-flow microreactor with a volume of less than $1 \mu 1$ is presented. This homemade microreactor is combined with a commercially available integrated circuit calorimeter. A similar approach was reported by Köhler and Zieren [3] and by Lerchner et al. [4-6]. Lerchner et al. investigated many different constructions of IC calorimeter [5] (batch and flow through calorimeter) well adapted to work with enzyme-catalyzed reactions. A new construction will be described here to extend the use of these systems to solve chemical safety issues and in particular for fast exothermic reaction kinetics in the liquid phase. The material used has to be chemically inert and the microreactor should be easily changed and adapted to the different reactions analyzed. 


\section{Nomenclature}

\begin{tabular}{|l|l|}
\hline$A$ & area $\left(\mathrm{m}^{2}\right)$ \\
\hline$B o$ & Bodenstein number $(-)$ \\
\hline$c$ & concentration $\left(\mathrm{mol}^{-1}\right)$ \\
\hline$C_{\mathrm{p}}$ & heat capacity $\left(\mathrm{J} \mathrm{kg}^{-1} \mathrm{~K}^{-1}\right)$ \\
\hline$d$ & diameter of the channel $(\mathrm{m})$ \\
\hline$d_{\mathrm{C}}$ & hydraulic diameter of the channel (m) \\
\hline$D_{\mathrm{ax}}$ & axial diffusion $\left(\mathrm{m}^{2} \mathrm{~s}^{-1}\right)$ \\
\hline$D_{\mathrm{m}}$ & molecular diffusion $\left(\mathrm{m}^{2} \mathrm{~s}^{-1}\right)$ \\
\hline$e$ & depth of the channel (m) \\
\hline$F$ & correction factor with the calibration $(\mathrm{m})$ \\
\hline$H_{\mathrm{R}}$ & heat of reaction $\left(\mathrm{J} \mathrm{mol}^{-1}\right)$ \\
\hline$J$ & matter flow (mol $\left.{ }^{-2} \mathrm{~s}^{-1}\right)$ \\
\hline$L$ & length of the channels (m) \\
\hline
\end{tabular}

\begin{tabular}{|l|l|}
\hline$m$ & mass $(\mathrm{kg})$ \\
\hline$\dot{q}$ & heat flow $(\mathrm{W})$ \\
\hline$r_{C}$ & hydraulic radius of the channel $(\mathrm{m})$ \\
\hline$R$ & resistance $\left(\mathrm{K} \mathrm{W}^{-1}\right)$ \\
\hline$S_{\mathrm{q}}$ & sensitivity $\left(\mu \mathrm{V} \mathrm{mW}^{-1}\right)$ \\
\hline$t_{\mathrm{D}}$ & time for radial diffusion $(\mathrm{s})$ \\
\hline$u$ & velocity $\left(\mathrm{m} \mathrm{s}^{-1}\right)$ \\
\hline$U$ & output voltage $(\mu \mathrm{V})$ \\
\hline$\dot{V}$ & flow rate $\left(\mathrm{m}^{3} \mathrm{~s}^{-1}\right)$ \\
\hline$X$ & conversion $(-)$ \\
\hline$\varepsilon$ & Seebeck coefficient $\left(\mathrm{V} \mathrm{K}^{-1}\right)$ \\
\hline$\lambda$ & thermal conductivity $\left(\mathrm{W} \mathrm{m}^{-1} \mathrm{~K}^{-1}\right)$ \\
\hline & \\
\hline
\end{tabular}

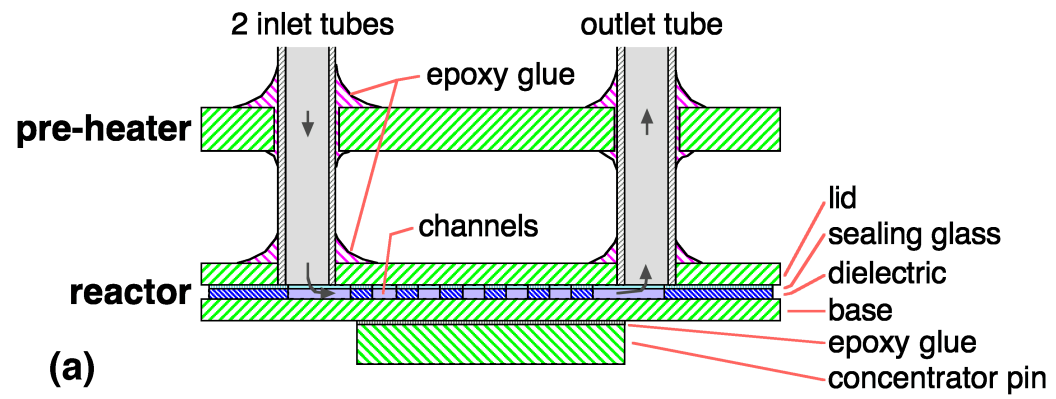

(b)

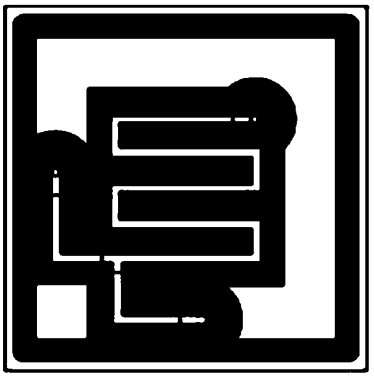

Fig. 1. (a) Lateral view of the microreactor and (b) top view of the reaction channel.

\section{Experimental}

\subsection{Microreactor design.}

The following steps were used to build the microreactors schematically shown in Fig. 1. First, the channel walls were created on the alumina base by screen-printing and firing of a commercial thick-film dielectric (ESL 4913, Agmet Limited, UK). These steps were repeated several times until the desired channel height (close to $100 \mu \mathrm{m}$ ) was reached. Each layer of dielectric gave a fired thickness of about $15 \mu \mathrm{m}$. In order to achieve chemical inertia and good heat conduction properties, the base was made of an alumina layer of $250 \mu \mathrm{m}$. On top of the dielectric, two layers of sealing glass (ESL 4026A, Agmet Limited, UK) were applied and pre-fired, giving about $20 \mu \mathrm{m}$ of seal thickness. The alumina lid was prepared by laser cutting of the inlet and outlet holes, and then sealed to the base using the sealing glass. After sealing, an alumina contact pin was glued on the bottom of the base. This was followed by gluing stainless steel tubes (AISI 316L, i.d.: $0.65 \mathrm{~mm}$, o.d.: $1 \mathrm{~mm}$, manufactured by Unimed, Switzerland) in the inlet and outlet holes, using an epoxy resin (Emerson and Cuming, Stycast 2651MM with catalyst 24LV). Finally, an aluminum screen was glued to the tube at a fixed position. 


\subsection{Layout of the calorimetric system}

The microreactor was incorporated into a commercially available microcalorimeter (Setline 120, Setaram, France). The IC used is the LCM 2524 (manufactured by Xensor Integration, The Netherlands). The IC has a total area of $72 \mathrm{~mm}^{2}$ but the calorimetric sensitive area is a $4 \mathrm{~mm} \times 4 \mathrm{~mm}$ square in the center of the chip $[4,5]$. A thermopile measures the temperature difference between the center of the membrane and the reference temperature of the rim.

The base of the microreactor conducts the heat released by the reaction in the channel towards the sensitive part of the calorimetric chip by an alumina contact pin glued under the lower side of the microreactor (Fig. 2). A small amount of heat carrier (Polyethylene glycol 300, Fluka, Switzerland) is introduced on the calorimetric chip to avoid thermally insulating air layers between the contact pin and the calorimetric chip.

The membrane of the IC is $45 \mu \mathrm{m}$ thick and is very fragile. An aluminum screen slightly larger than the border of the oven cavity is glued to the reactor tubes using a reference jig to precisely define its vertical position. This allows a reproducible position near to the membrane. This screen also permits the reflection of the heat into the measurement cavity and the preheating of the tubes.

A syringe pump (Bioblock Scientific KDS 200, Ismatec, Switzerland) with two Hamilton glass syringes (Hamilton, Verrerie de Carouge, Switzerland) is used for the control of liquid flow rates.

\subsection{Assessment of the degree of mixing in the reaction channel}

The iodide-iodate reaction system [7-13] was used to determine the degree of mixing in the microchannels.

This method is based on a competitive parallel reaction system:

$$
\begin{aligned}
& \mathrm{H}_{2} \mathrm{BO}_{3}{ }^{-}+\mathrm{H}^{+} \rightleftarrows \mathrm{H}_{3} \mathrm{BO}_{3} \text { (quasi-instantaneous) } \\
& 5 \mathrm{I}^{-}+\mathrm{IO}_{3}{ }^{-}+6 \mathrm{H}^{+} \rightleftarrows 3 \mathrm{I}_{2}+3 \mathrm{H}_{2} \mathrm{O} \text { (very fast) }
\end{aligned}
$$

$\mathrm{H}^{+}$is added in a stoichiometric defect. In case of ideal mixing, $\mathrm{H}^{+}$will be totally consumed by the first reaction and there will be no $I_{2}$ observed. The concentration of $I_{2}$ is proportional to the segregation in the microchannel. The iodine then reacts quasi-instantaneously with iodide ions.

$$
\mathrm{I}_{2}+\mathrm{I}-\rightleftarrows \mathrm{I}_{3}^{-}
$$

The concentration of $\mathrm{I}_{3}^{-}$is measured by UV spectrometry at $352 \mathrm{~nm}$. As the $\mathrm{I}_{3}^{-}$formed is stable, the absorbance can be measured offline. An ideal mixing is characterized by an absorbance at $352 \mathrm{~nm}$ close to zero.

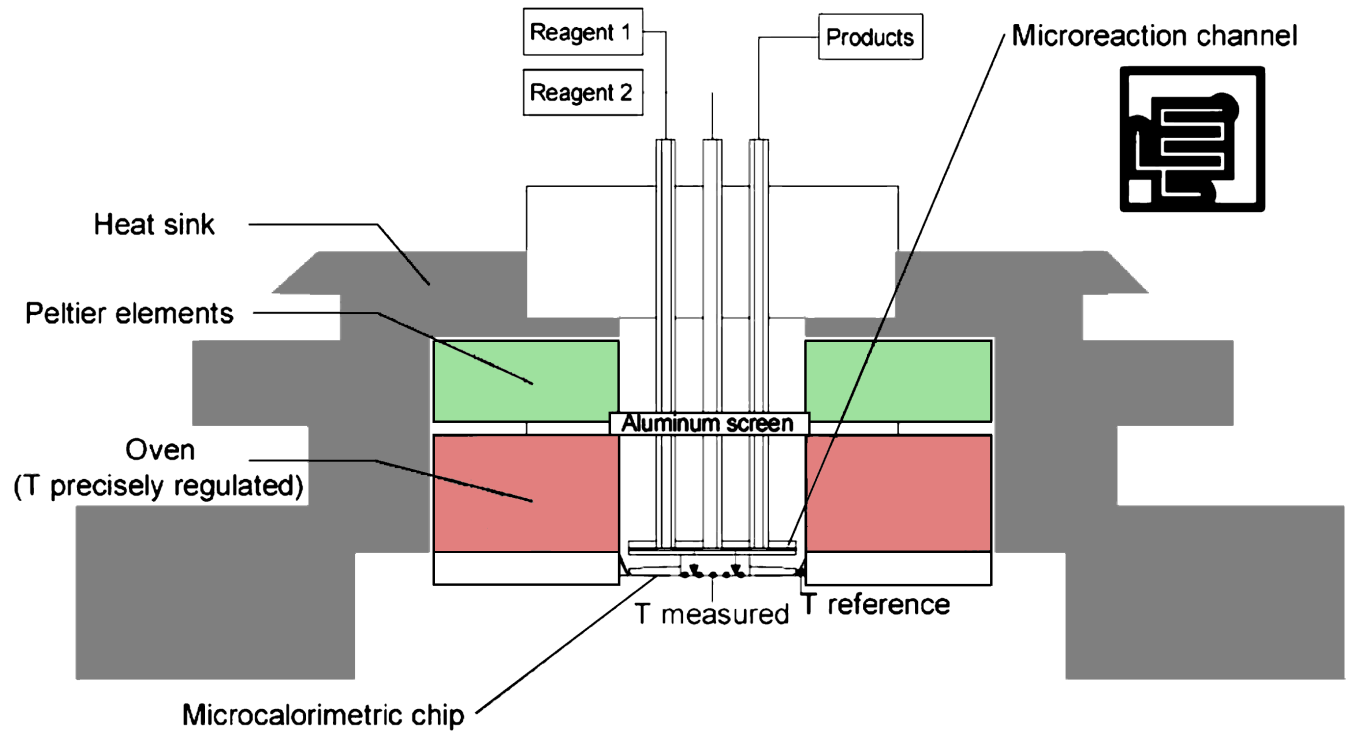

Fig. 2. Lateral view of the experimental layout. 
The concentrations were slightly increased to improve the sensitivity of this method in the small reaction channels. The following concentrations were therefore used: $c_{\mathrm{KIO} 3}=2.33 \times 10^{-3} \mathrm{~mol} \cdot 1^{-1}$, $c_{\mathrm{KI}}=1.66 \times 10^{-2} \mathrm{~mol} \cdot 1^{-1}, c_{\mathrm{NaOH}}=9.09 \times 10^{-2} \mathrm{~mol} \cdot \cdot^{-1}, c_{\mathrm{H} 3 \mathrm{BO} 3}=1.82 \times 10^{-1} \mathrm{~mol} \cdot \cdot^{-1}$ and $c_{\mathrm{H}+}=2 \times 10^{-2} \mathrm{~mol} \cdot \mathrm{l}^{-1}$. The inlet flow rates were varied from 3 to $19 \mu 1 \cdot \mathrm{min}^{-1}$. One syringe contained the solution of $\mathrm{KIO}_{3}, \mathrm{KI}, \mathrm{NaOH}$ and $\mathrm{H}_{3} \mathrm{BO}_{3}$; the other syringe contained the aqueous solution of sulphuric acid. A sample of $50 \mu 1$ was taken offline once steady state was achieved. The absorbance at $352 \mathrm{~nm}$ of the reaction product $\mathrm{I}_{3}{ }^{-}$was measured at each flow rate.

The absorbance measurements were done in a $50 \mu 1$ cuvette (HELLMA) with a UV spectrometer HP 8452 (Hewlett-Packard, USA).

\subsection{Calibration of the calorimetric system}

\subsubsection{Using the heater resistance}

A heater resistance incorporated in the middle of the sensitive area can be used for calibration. A Joule effect of $\dot{q}=1 \mathrm{~mW}$ is applied to the system for $60 \mathrm{~s}$ and the resulting output voltage $U$ measured by the thermopile is then integrated. The sensitivity of the system $S_{\mathrm{q}}$ can be calculated from the following equation:

$$
S_{\mathrm{q}}=U / \dot{q}
$$

This sensitivity is controlled and re-determined before each measurement by performing two impulses at $1 \mathrm{~mW}$. However, this sensitivity corresponds to the sensitivity of the chip and does not characterize the entire system.

\subsubsection{Using a chemical reaction}

In this calibration, the sensitivity of the entire system can be determined. The neutralization of $\mathrm{H}_{2} \mathrm{SO}_{4}$ by $\mathrm{NaOH}$ is carried out in the reaction channel and the corresponding signal is measured. For a continuousflow reactor, the global heat flow $\dot{q}_{\mathrm{rx}}$ released in the channel by the reaction is

$$
\dot{q}_{\mathrm{rx}}=1000 \dot{V} \cdot\left(-\Delta H_{\mathrm{R}}\right) \cdot\left(c_{0}-c_{\mathrm{f}}\right)=1000 \dot{V} \cdot\left(-\Delta H_{\mathrm{R}}\right) \cdot \mathrm{c}_{0} \cdot X
$$

The flow rate can be varied from 2 to $6 \mu 1 \cdot \mathrm{min}^{-1}$ and the concentration of the reagents is varied from 0 to $0.4 \mathrm{~mol} \cdot \mathrm{l}^{-1}$. The heat of reaction is well known and is $-130 \mathrm{~kJ} \mathrm{~mol}^{-1}$ [14]. As this neutralization reaction is quasi-instantaneous, the conversion in the channel can be considered to be complete. This experiment is performed for every change in the microsystem (microreactor, heat carrier layer, etc.).

\section{Results}

\subsection{Characteristics of the flow}

\subsubsection{Degree of mixing in the reaction channel}

The method of fabrication of the microreactor allows the realization of various geometries for the reaction channel design. The positions of the inlet and the outlet were maintained constant to make the connections easier. The length of the reaction channel can be varied and adapted to the needs of the reaction.

The degree of mixing in three different designs was assessed in order to evaluate the influence of the length and of the shape of the channel on the quality of mixing (see Fig. 3). As expected, it was observed that the degree of mixing increased with increasing length of the channel. The design 3 gave the best results and was used in the following experiments.

\subsubsection{Time for radial diffusion and Bodenstein number}

To determine kinetics parameters, the distribution of the residence times in the channel must be precisely known. The characterization of the flow was done in two ways, first by calculating the time of radial diffusion in the microchannel which gives an idea of the radial solute distribution and then by using the model of dispersion to quantify the degree of retromixing. 

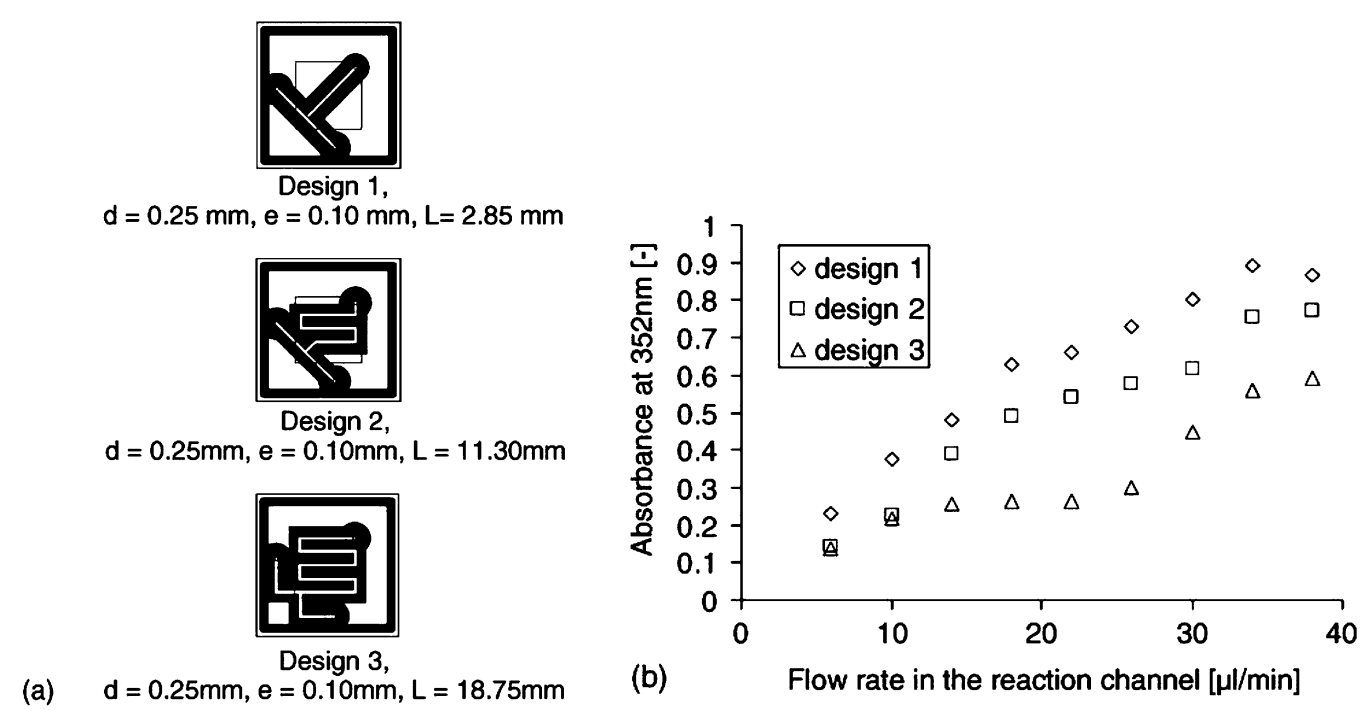

(b) Flow rate in the reaction channel $[\mu \mathrm{l} / \mathrm{min}]$

Fig. 3. Characterization of the degree of mixing: (a) scheme of the three designs tested, $d, L$ and $e$ are respectively the width, the length and the depth of the channel; (b) absorbance at $352 \mathrm{~nm}$ obtained for each design using the iodide-iodate reaction system.

\subsubsection{Time for radial diffusion [15]}

The time required for a complete radial diffusion $t_{\mathrm{D}}$ can be calculated from

$$
t_{\mathrm{D}}=r_{\mathrm{C}}^{2} / D_{\mathrm{m}}
$$

where $r_{\mathrm{C}}$ is the hydraulic diameter and is equal to $89.2 \mu \mathrm{m}$ and the molecular diffusion, $D_{\mathrm{m}}$, is equal to $1 \times 10^{-8} \mathrm{~m}^{2} \cdot \mathrm{s}^{-1}$ in the case of protons; $t_{\mathrm{D}}$ is then $0.8 \mathrm{~s}$.

\subsubsection{Bodenstein number}

The Bodenstein number $(B o)$ characterizes the ratio between the convection in the channel and the axial diffusion.

$$
B o=\left(u / D_{\mathrm{ax}}\right) \cdot L
$$

where $L$ is the length of the channel, $u$ the velocity and $D_{\text {ax }}$ the axial diffusion in the channel. $B o$ tends to infinity for an ideal tubular reactor and to zero if the retromixing is very high. The axial diffusion and the Bodenstein number were calculated according to Ref. [16].

As shown in Fig. 4, Bo exceeds 300 for the entire range of flow rates currently used.

\subsection{Experimental characterization of the calorimetric system}

\subsubsection{Influence of the polyethylene glycol layer (PEG 300) and of the contact pin}

The neutralization of $\mathrm{H}_{2} \mathrm{SO}_{4}$ by $\mathrm{NaOH}$ was performed in the channel and the influence of the introduction of the contact pin and of the heat carrier layer on the calorimetric signal was assessed.

The oven temperature was set to $35^{\circ} \mathrm{C}$. Heat flows measured experimentally with initial concentrations of $\mathrm{NaOH}$ and $\mathrm{H}_{2} \mathrm{SO}_{4}$ varying from 0.1 to $0.4 \mathrm{~mol} \cdot \mathrm{l}^{-1}$ at a constant inlet flow rate of $5 \mu 1 \cdot \mathrm{min}^{-1}$ were compared to the heat released in the channels as calculated using Eq. (2). Water at a flow rate of $5 \mu 1 \cdot \mathrm{min}^{-1}$ was used as a baseline.

As shown in Fig. 5, the introduction of the microreactor in the cavity of the microcalorimeter without any adjustment translated into only $10 \%$ of the expected signal. This ratio increased to $21 \%$ when a heat carrier layer (PEG 300) was introduced between the calorimetric chip and the microreactor and finally reached $53 \%$ of the heat released in the channel with the addition of a contact pin. The results obtained by varying the flow rates at a given concentration were comparable (results not shown). 


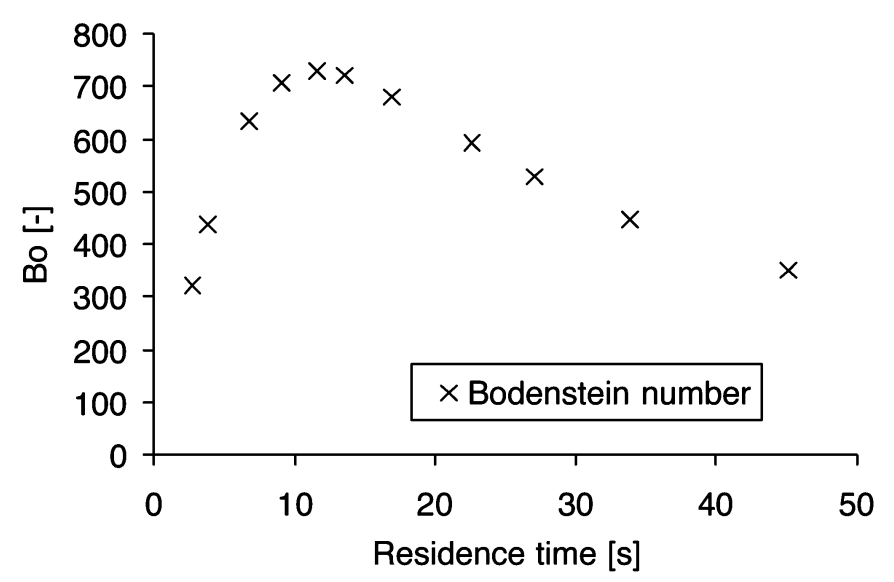

Fig. 4. Variation of the Bodenstein number with the residence time in the reaction channel.
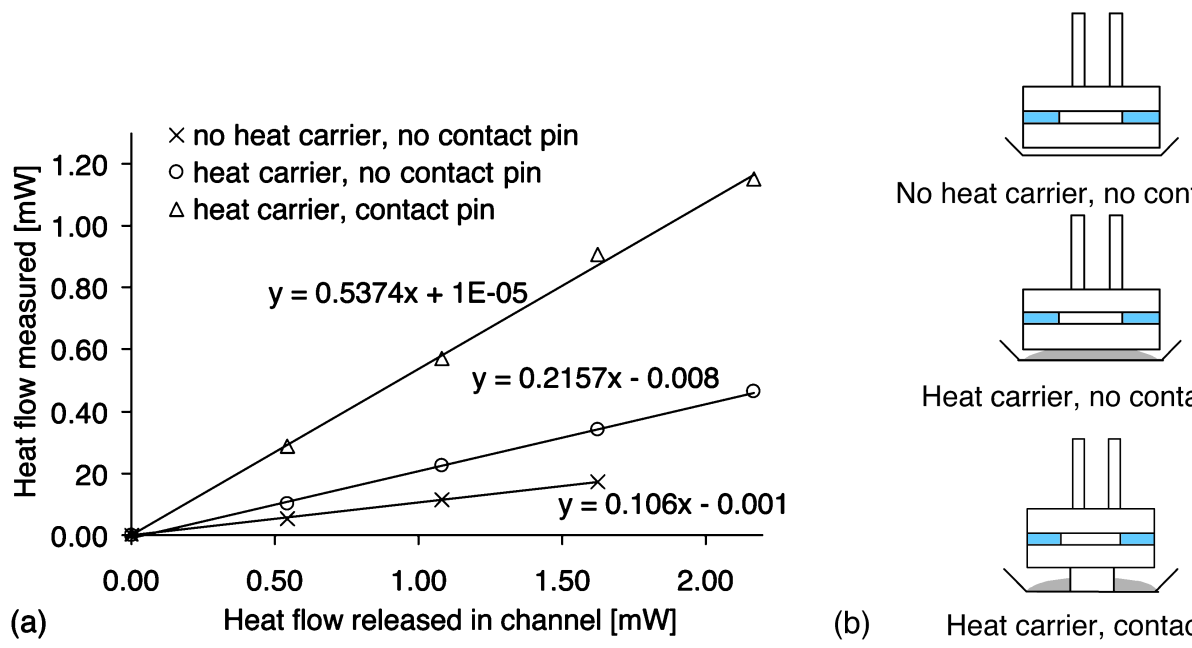

No heat carrier, no contact pin

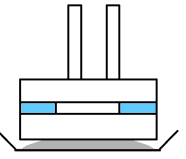

Heat carrier, no contact pin

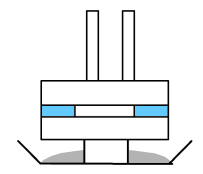

(b) Heat carrier, contact pin

Fig. 5. (a) Influence of the heat carrier and of the contact pin on the heat measured when a neutralization reaction is performed in the channel; (b) scheme of the three tested configuration.

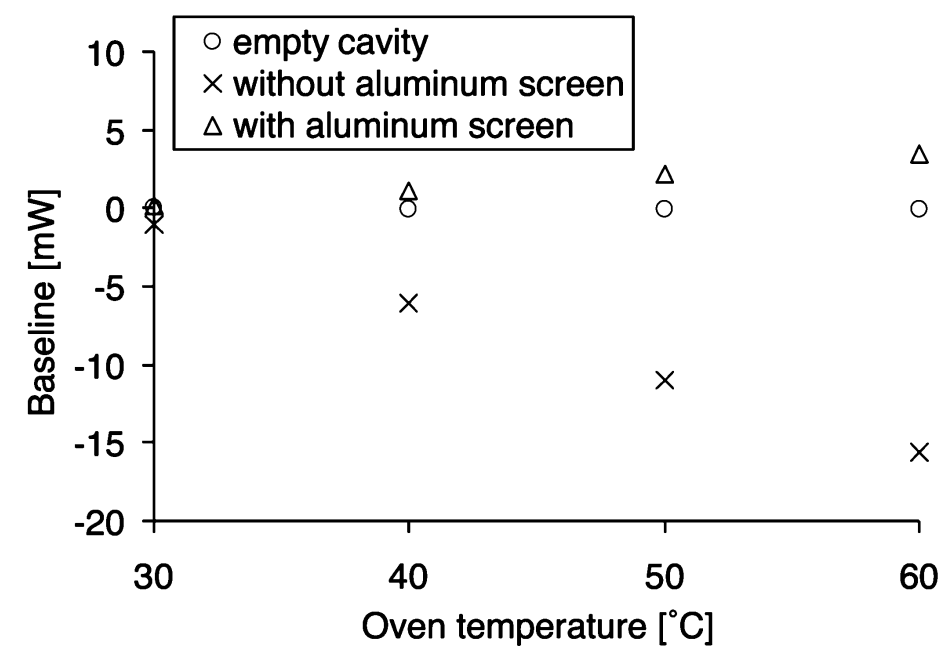

Fig. 6. Influence of the aluminum screen on the position of the baseline at different oven temperatures. 


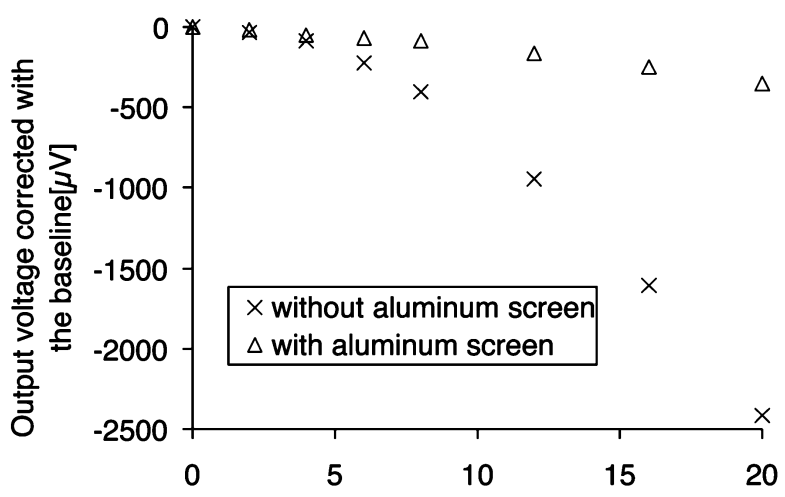

(a)

Flow rate total $[\mu \mathrm{l} / \mathrm{min}]$

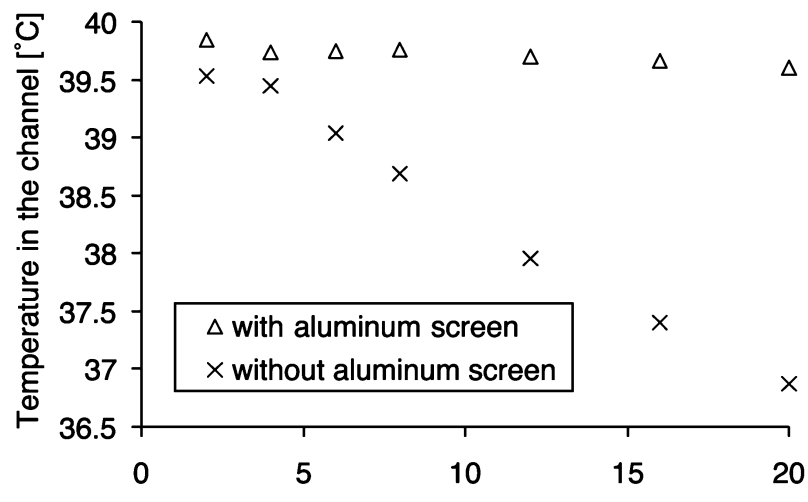

(b)

Flow rate total $[\mu \mathrm{l} / \mathrm{min}]$

Fig. 7. Influence of the aluminum screen on the preheating of the entering fluid at $25 \circ \mathrm{C}$ in the oven at $40 \circ \mathrm{C}$ : (a) effect on the output voltage; (b) temperature achieved in the reaction channel calculated from the output voltage measurement.

The sensitivity of the calorimeter is highly dependent on the amount of heat carrier. However, polyethylene glycol is difficult to dose precisely because of its high viscosity. The ratio can be improved to more than $60 \%$ with proper adjustment of the amount of heat carrier. A calibration should therefore be performed every time the heat carrier layer is modified and thus every time the microreactor is changed or moved.

\subsubsection{Influence of the aluminum screen}

The influence of the aluminum screen on the heat flow was tested by varying the temperature of the oven. At steady state, no signal is expected and the baseline should be close to zero. However, if the temperature of the microreactor never reaches the oven temperature, an endothermic signal will be observed.

As seen in Fig. 6, without aluminum screen, the temperature of the microreactor never reached the oven temperature and the baseline was negative. However, with the aluminum screen, a slightly exothermic signal was observed.

The preheating of the entering fluid was then assessed by introducing water at $25^{\circ} \mathrm{C}$ in the microreactor. The oven temperature was set at $40^{\circ} \mathrm{C}$. If the entering fluid is not at the oven temperature, a sensible heat will be measured and an endothermic signal will be observed. As seen in Fig. 7, the aluminum screen improved the preheating of the entering fluid.

\subsection{Repeatability and reproducibility}

\subsubsection{Repeatability}

The oven temperature was set at $35^{\circ} \mathrm{C}$. The microreactor was not changed or moved during the time of experiment. Nine experiments were carried out varying the concentration of $\mathrm{NaOH}$ and $\mathrm{H}_{2} \mathrm{SO}_{4}(0,0.1,0.2$, 0.3 and $\left.0.4 \mathrm{~mol} \cdot 1^{-1}\right)$ and varying the flow rates $\left(2,4\right.$ and $\left.6 \mu 1 \cdot \mathrm{min}^{-1}\right)$. The series of experiments was repeated over a week to assess the repeatability in time. In a total of nine experiments, an average deviation of $3 \%$ was observed (see Fig. 8). No variations with time were observed.

\subsubsection{Reproducibility}

The reproducibility was tested by changing the neutralization reaction. The neutralization of $\mathrm{HCl}$ by $\mathrm{NaOH}$ was carried out and compared to the results of the neutralization of $\mathrm{H}_{2} \mathrm{SO}_{4}$ by $\mathrm{NaOH}$. The same sensitivity was observed with both reactions. A typical result is shown in Fig. 9. 


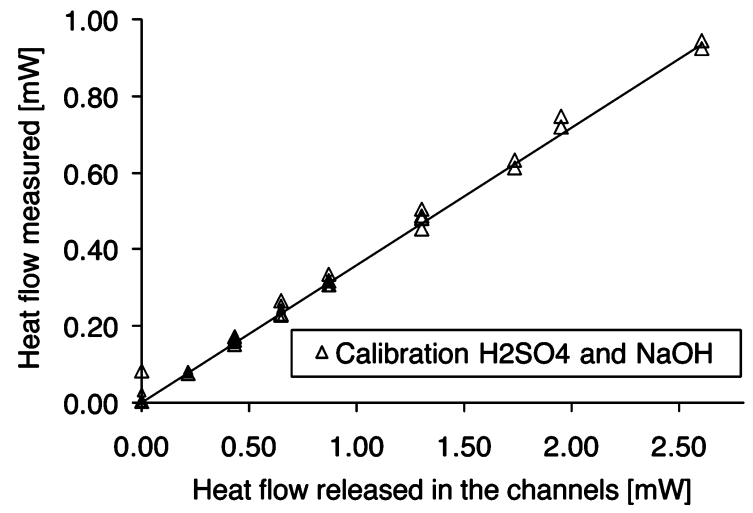

Fig. 8. Repeatability of the measurements.

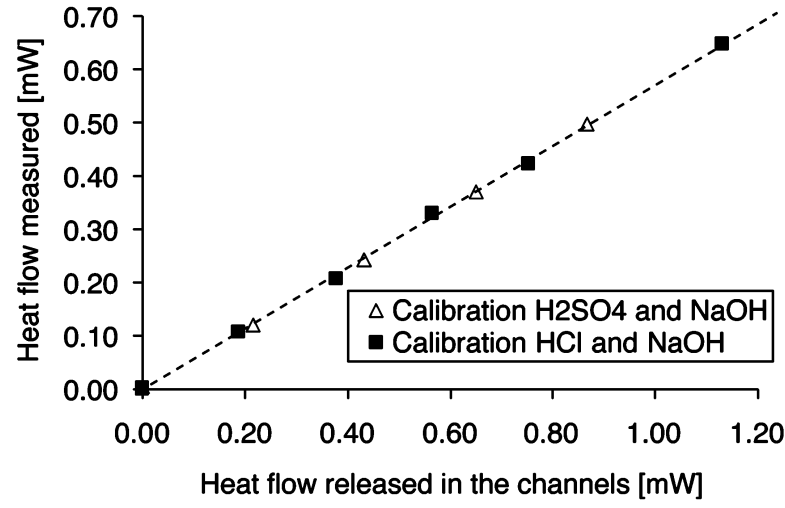

Fig. 9. Reproducibility of the measurements.

\subsection{Detailed characterization of the calorimetric system: heat flow balance}

For a better understanding of the calorimetric system, a heat flow balance was established allowing the evaluation of the individual contribution of the different parts of the system. The heat released in the channel can be distributed in four main ways:

- in the sensitive part of the IC membrane;

- in the non-sensitive part of the IC membrane;

- in the stainless steel tubes or in the air above the reactor;

- as heat accumulation in the outlet flow.

Three resistances can be defined to achieve a better description of the system (Fig. 10):

- $R_{\mathrm{IC}}$ : the resistance of the integrated circuit (membrane).

- $R_{\text {contact: }}$ the resistance due to the heat transfer from the reaction channel to the sensitive zone.

- $R_{\text {loss: }}$ : the resistance due to the various losses (air convection, heat accumulation in the outlet flow and by heat conduction in the stainless steel tube and in the non-sensitive part of the membrane).

To estimate these resistances, two calibrations described in Section 2.4 were used. The calculations are detailed in Appendix A but the most important formulas are summarized in the next paragraphs.

\subsubsection{Heat source on the chips: calibration using the heater resistance}

A Joule effect was applied at the center of the membrane first with an empty cavity and then with the microreactor introduced in the cavity. The respective sensitivities $S_{\mathrm{q} 0}$ and $S_{\mathrm{q}}^{\prime}$ were determined. The ratio of these sensitivities can be expressed as following:

$$
S_{\mathrm{q}}^{\prime} / S_{\mathrm{q} 0}=\left(R_{\text {contact }}+R_{\text {loss }}\right) /\left(R_{\mathrm{IC}}+R_{\text {contact }}+R_{\text {loss }}\right)
$$

The sensitivity obtained was $1500 \mu \mathrm{V} \cdot \mathrm{mW}^{-1}$ for the empty cavity $\left(S_{\mathrm{q} 0}\right)$ and $1080 \mu \mathrm{V} \cdot \mathrm{mW}^{-1}$ for the entire system $\left(S_{\mathrm{q}}^{\prime}\right)$ (Fig. 11).

The resistance of the integrated circuit $R_{\mathrm{IC}}$ can be calculated from the sensitivity of the empty cavity using Eq. (A.10) in Appendix A and is equal to $21.4 \mathrm{~K} \cdot \mathrm{W}^{-1}$.

The sum $\left(R_{\text {loss }}+R_{\text {contact }}\right)$ can then be estimated from Eq. (5) and is equal to $56 \mathrm{~K} \cdot \mathrm{W}^{-1}$. 


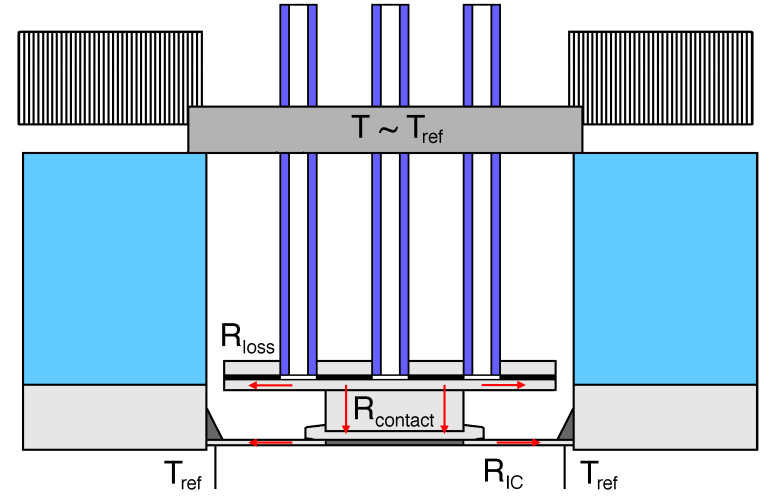

Fig. 10. Drawing of the calorimetric cavity. Heat flow balance.

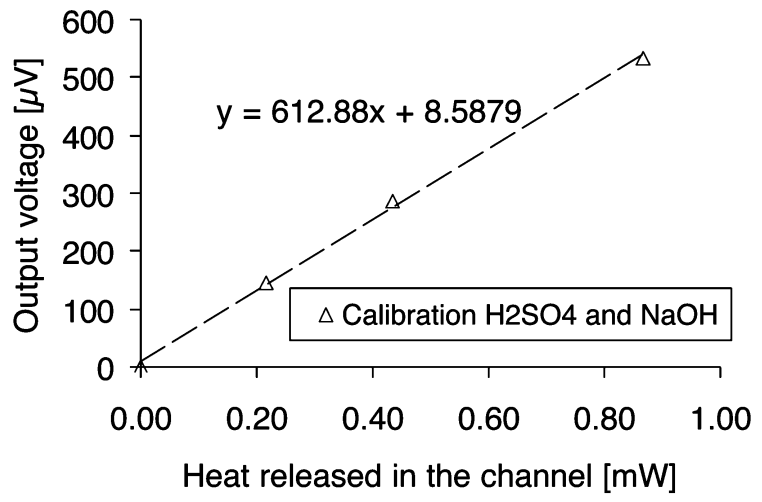

Fig. 12. Determination of the sensitivity of the system for a heat source in the reaction channel.

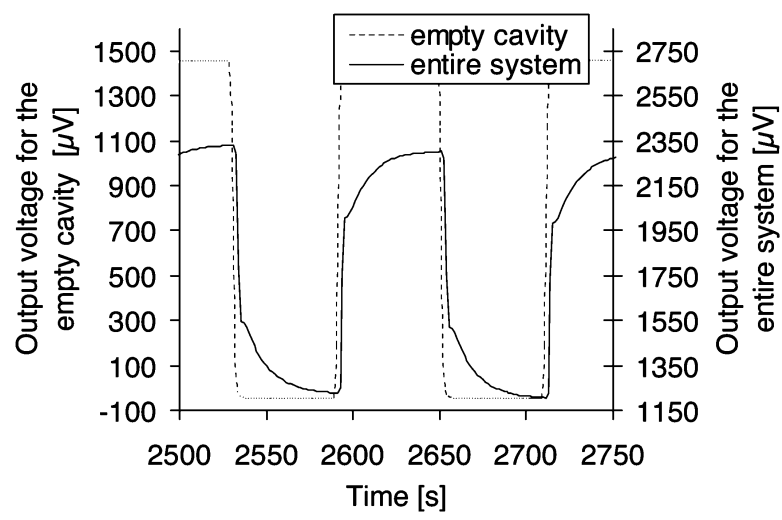

Fig. 11. Comparison of the sensitivity with and without microreactor in the calorimetric cavity.

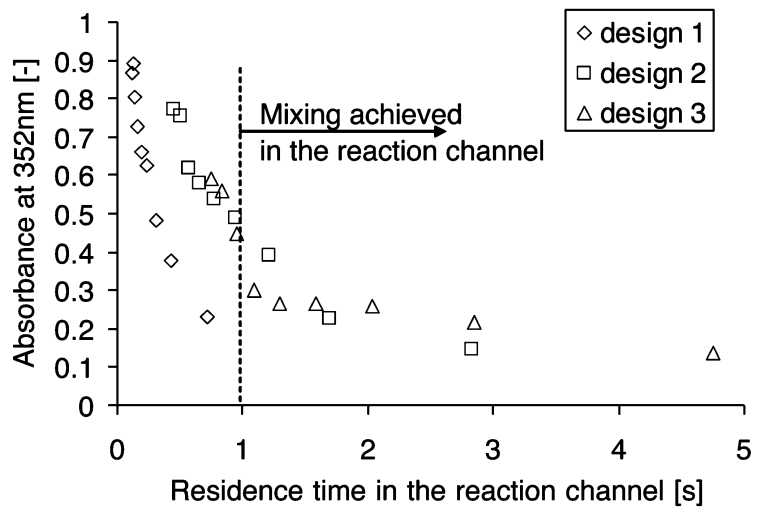

Fig. 13. Absorbance at $352 \mathrm{~nm}$ obtained in three different designs according to the residence time in the reaction channel.

\subsubsection{Heat source in the reaction channel: calibration using a chemical reaction}

In this case, the neutralization of $\mathrm{H}_{2} \mathrm{SO}_{4}$ by $\mathrm{NaOH}$ is performed in the channels. The sensitivity obtained in this case $S^{\prime \prime}{ }_{\mathrm{q}}$ can be compared to the previous one $S_{\mathrm{q}}^{\prime}$ :

$$
S^{\prime \prime}{ }_{\mathrm{q}} / S_{\mathrm{q}}=R_{\text {loss }} /\left(R_{\text {contact }}+R_{\text {loss }}\right)
$$

Knowing the sum $\left(R_{\text {loss }}+R_{\text {contact }}\right)$ from the first calculation, $R_{\text {loss }}$ can be estimated and thus compared to $R_{\text {contact. }}$

An experiment similar to the one performed in Section 3.2 was repeated and the corresponding output voltage was measured (Fig. 12). For this experiment, the inlet flow rates used were $2 \mu 1 \cdot \mathrm{min}^{-1}$.

The sensitivity determined by this method was $612 \mu \mathrm{V} \cdot \mathrm{mW}^{-1}$. The value of the resistance of contact and of the resistance of losses can be deduced from Eq. (6), giving $R_{\text {contact }}=24 \mathrm{~K} \cdot \mathrm{W}^{-1}$ and $R_{\text {loss }}=32 \mathrm{~K} \cdot \mathrm{W}^{-1}$. 


\section{Discussion}

\subsection{Characteristics of the flow in the reaction channel}

\subsubsection{Influence of the flow rate on the degree of mixing}

As shown in Fig. $3 b$ the degree of mixing decreases when the flow rate in the reaction channel increases. This tendency was also observed by Floyd et al. [2]. Floyd et al. measured the degree of mixing using two phenol red streams at $\mathrm{pH}$ values 6.4 and 8.0, and measured the absorbance online at the end of the channel. At low flow rates, the absorbance dropped because the solution had a $\mathrm{pH}$ close to 7.2. The inverse tendency was reported by Ehrfeld et al. [17] using the iodide-iodate system but this was explained by the very high flow rate used (more than 1000 times higher than the ones used in our layout). In this case, the slow formation of iodine was only observed at low flow rates and the absorbance increased as the residence time increased.

\subsubsection{Molecular diffusion in the reaction channel}

In our case, even at a flow rate of $40 \mu 1 \cdot \mathrm{min}^{-1}$, the Reynolds number is lower than 8 and the flow is clearly laminar. The mixing is therefore only achieved by molecular diffusion. When the flow rate increases, the contact time in the reaction channel decreases, and as molecular diffusion is a slow phenomenon, homogeneity is not obtained at the end of the reaction channel. The absorbance values obtained in Fig. $3 \mathrm{~b}$ for the three designs tested can be plotted against the residence time in the channel (see Fig. 13).

For residence times above $1 \mathrm{~s}$, the mixing is independent of the flow rate as expected for a fully laminar system [17]. However, for smaller residence times, the degree of mixing becomes highly dependent on the flow rate and decreases strongly with increasing flow rates. Mixing is not achieved in the reaction channel and takes place in the outlet tube. As the outlet tube has a larger diameter than the reaction channel, more segregation is observed.

\subsubsection{Time for radial diffusion}

These results are confirmed by the calculation of the time for radial diffusion. As seen in Section 3.1, the time required for radial diffusion is $0.8 \mathrm{~s}$ in the case of protons. For residence times shorter than $0.8 \mathrm{~s}$, mixing is then achieved in the outlet tube.

In the three layouts tested, the shape of the reaction channel does not significantly improve the mixing. However, a sufficient length of the channel is necessary to guarantee that the mixing takes place entirely in the reaction channel. For the determination of reaction kinetics using the microcalorimetric system, the time required for mixing should be short compared to the residence time in the channel so that the heat measured can be related to the conversion rate of the reaction. In our case, the flow rates currently used for kinetics determinations were between 0.3 and $1 \mu 1 \cdot \mathrm{min}^{-1}$ and the residence times were between 13 and $45 \mathrm{~s}$. The time for radial diffusion was always lower than $6 \%$ of the residence time, and thus the error on the kinetics should be low. Smaller diameters will probably be needed when working with substances with a lower molecular diffusion than protons.

\subsubsection{Bodenstein number}

The calculation of the Bodenstein number showed that the retromixing was negligible in the range of flow rates currently used. The reactor behaved like a plug flow reactor. There is no distribution of the residence time. This is also an important point for the determination of reaction kinetics.

\subsection{Characterization of the calorimetric system}

\subsubsection{Heat carrier layer and contact pin}

As seen in Fig. 5, the introduction of the heat carrier and of the contact pin improves the sensitivity of the microsystem by a factor of 5 . However, even with a heat carrier layer and a contact pin, the heat released in the channel is still not entirely directed to the sensitive area of the membrane. A maximum of $60 \%$ of the expected signal was measured. This ratio was independent of the flow rate and of the concentration of the reagent (see Fig. 8) which is a fundamental point for the determination of kinetics. This ratio was called "efficiency" by Köhler and Zieren [3]. The efficiency obtained in the microsystem developed by Köhler had a maximum of $70 \%$ but was dependent on the flow rate used. For flow rates higher than $5 \mu 1 \cdot \mathrm{min}^{-1}$ the efficiency dropped to $30 \%$. 
The maximum of $60 \%$ observed in our system can be explained in the following way. The commercially available microcalorimeter Setline 120 is made of two parts. The upper part of the calorimeter contains the oven and the lower part contains the calorimetric membrane. When the calorimeter is closed, the upper part is fixed on the lower part with three screws. The contact pin is centered in relation to the oven but few millimeters of play in this fixation is enough to move the contact pin away from the center of the membrane. Part of the signal is therefore lost in the non-sensitive part of the membrane. This was confirmed by applying a paste on the surface of the contact pin and closing the calorimeter. When the calorimeter was opened, the mark of the position of the contact pin was slightly on the left hand side of the sensitive area. The exact positioning of the contact pin is difficult to control and a further optimization of the design of the system will have to be performed.

However, as long as the efficiency is repeatable and reproducible (see Figs. 8 and 9) the amount of heat lost in the non-sensitive part of the system is not a major issue.

\subsubsection{Aluminum screen}

As seen in Fig. 6, the introduction of the aluminum screen has a major effect on the temperature of the microreactor. Actually, the temperature of the microreactor is even slightly higher than the oven temperature. This can be explained by an inhomogeneous distribution of the temperature in the oven. The upper side of the oven which is directly in contact with the Peltier elements seems to be at a slightly higher temperature than the lower side in contact with the reference. This temperature difference can be estimated as $0.05^{\circ} \mathrm{C}$ at the highest temperature tested $\left(60^{\circ} \mathrm{C}\right)$ and will not influence the determination of kinetics. However, work is in progress in order to obtain a baseline independent of the temperature and close to zero.

The introduction of the aluminum screen, in which the inlet tubes are glued, induced a very efficient preheating of the entering fluids (see Fig. 7) even at a flow rate of $20 \mu 1 \cdot \mathrm{min}^{-1}$ (10 times higher than the current flow rate used). This is an important advantage of this system since entering fluids are easily heated to the reaction temperature. Obviously, the range of usable flow rates will depend on the temperature needed.

\subsubsection{Heat balance flow}

The three following observations can be made concerning the values of $R_{\text {contact }}$ and $R_{\text {loss }}$. Firstly, the resistance of contact is lower but comparable to the resistance of losses. In an ideal case, the resistance of losses should tend to infinity and the resistance of contact to zero. The low value of $R_{\text {loss }}$ is due to heat transfer in the non-sensitive part of the membrane as discussed in Section 4.2.1.

Secondly, the linearity of Figs. 8, 9 and 12 shows that there is no variation of the resistance of losses with the flow rates and the concentrations. The heat accumulation in the outlet tube can thus be neglected. Isothermal conditions are achieved even with the higher concentrations and the higher flow rates tested.

Lastly, the point of contact will be the limiting part for the rate of heat transfer. As a first approximation for the time for thermal diffusion in this point, the thermal diffusion in the polyethylene glycol layer can be estimated.

The resistance in the polyethylene glycol layer can be evaluated as follows:

$$
R_{\text {heat carrier }}=d /(\lambda \cdot A)
$$

Assuming that the height of the PEG 300 layer is $0.1 \mathrm{~mm}$, the area $16 \mathrm{~mm}_{2}$ and knowing that the conductivity of the polyethylene glycol is $0.23 \mathrm{~W} \cdot \mathrm{m}^{-1} \cdot \mathrm{K}^{-1}$, the resistance is therefore $27 \mathrm{~K} \cdot \mathrm{W}^{-1}$ (which is near to the experimental value of $24 \mathrm{~K} \cdot \mathrm{W}^{-1}$ for the resistance of contact). The time constant for thermal diffusion in this layer can thus be roughly estimated as

$$
t=\mathrm{R}_{\text {heat carrier }} \cdot m \cdot C_{\mathrm{p}}
$$

Knowing that $C_{\mathrm{p}}$ is $2 \mathrm{~kJ} \cdot \mathrm{kg}^{-1} \cdot \mathrm{K}^{-1}$ and assuming that the mass of PEG is $0.12 \mathrm{~g}(100 \mu \mathrm{l})$, the time constant is close to $7 \mathrm{~s}$. This time is much longer than the time required for the lateral distribution in the alumina plate of the microreactor (estimated using the same method as $50 \mathrm{~ms}$ ); the position of the reaction channel is therefore not limited to the area of the contact pin. The time for thermal diffusion is also much higher than the time for the lateral distribution in the membrane (around $10 \mathrm{~ms}$ ).

This will however not be a limiting point since all measurements are done at steady state. 


\section{Conclusions}

The new system developed showed a good calorimetric potential. The width to length ratio of the channel allowed a good mass transfer and a satisfactory degree of mixing; the calculated time for complete mixing $(0.8 \mathrm{~s})$ was confirmed experimentally. The length of the reaction channel was adapted to assure that this time of mixing is short compared to the residence time in the reaction channel.

The microreactors were designed to improve the sensitivity of the system and the resistances to the heat transfer were identified and characterized. The calibration of the system showed repeatable and reproducible results in the current range of flow rates and of concentrations used and a good preheating of the fluids was achieved before their entrance in the reaction channel.

It was shown that, even after some optimizations, the limiting part for the heat transfer was still the contact between the microreactor and the calorimetric membrane. This could be further improved in a future work.

\section{Acknowledgements}

Grateful acknowledgements are made to G. Corradini and C. Jacq for the realization of the microreactors.

\section{Appendix A. Detailed calculations of the heat flow balance}

\section{A.1. Heat source on the chips: calibration using the heater resistance}

Applying a Joule effect of $\dot{q}=1 \mathrm{~mW}$, the sensitivity is defined as

$$
S_{\mathrm{q}}=U_{\mathrm{m}} / \dot{q}
$$

\section{A.1.1. Empty cavity}

Without microreactor, the heat flow $\dot{q}$ can be divided into two parts:

$$
\dot{q}=\dot{q}_{\mathrm{m} 0}+\dot{q}_{\mathrm{loss}}
$$

where $\dot{q}_{\text {loss }}$ is the heat flow lost equal to

$$
\dot{q}_{\text {loss }}=1 / R_{\text {air }} \cdot \Delta T
$$

and $\dot{q}_{\mathrm{m} 0}$ is the heat flow measured by the IC membrane:

$$
\dot{q}_{\mathrm{m} 0}=1 / R_{\mathrm{IC}} \cdot \Delta T
$$

Because the resistance of the air is high compared to the resistance of the integrated circuit, Eq. (A.2) can be simplified into

$$
\dot{q}_{\mathrm{m} 0}=\dot{q}
$$

\section{A.1.2. Entire system}

With the microreactor and the heat carrier layer, the contribution of the resistances can be approximated as in Fig. 14. In this case, the heat flow lost is equal to

$$
\dot{q}^{\prime} \text { loss }=1 /\left(R_{\text {contact }}+R_{\text {loss }}\right) \cdot \Delta T
$$

The expression of the heat flow measured, $\dot{q}^{\prime}$ m , then becomes

$$
\dot{q}_{\mathrm{m}}^{\prime}=\dot{q} \cdot\left(R_{\text {contact }}+R_{\text {loss }}\right) /\left(R_{\mathrm{IC}}+R_{\text {contact }}+R_{\text {loss }}\right)
$$

Combining Eqs. (A.5) and (A.7), we obtain

$$
\dot{q}_{\mathrm{m}}^{\prime} / \dot{q}_{\mathrm{m} 0}=U_{\mathrm{m}}^{\prime} / U_{\mathrm{m} 0}=\dot{q} / \dot{q} \cdot\left(R_{\text {contact }}+R_{\text {loss }}\right) /\left(R_{\mathrm{IC}}+R_{\text {contact }}+R_{\text {loss }}\right)
$$

The ratio of the measured sensitivities is then

$$
S_{\mathrm{q}}^{\prime} / S_{\mathrm{q} 0}=\left(U_{\mathrm{m}}^{\prime} / \dot{q}\right) /\left(U_{\mathrm{m} 0} / \dot{q}\right)=\left(R_{\text {contact }}+R_{\text {loss }}\right) /\left(R_{\mathrm{IC}}+R_{\text {contact }}+R_{\text {loss }}\right)
$$




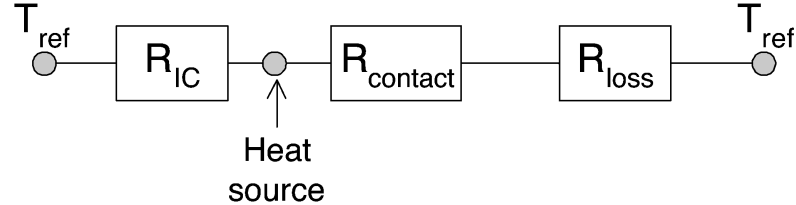

Fig. 14. Scheme of the resistance contributions for the entire system with a heat source on the chip.

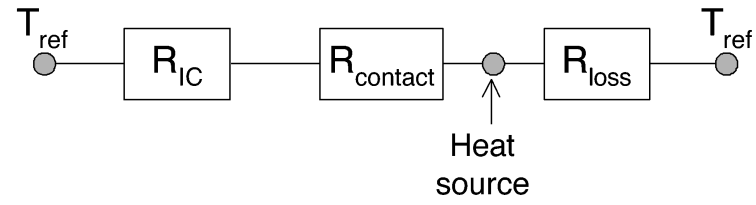

Fig. 15. Scheme of the resistance contributions for the entire system with a heat source in the channel.

$R_{\mathrm{IC}}$ can be estimated by the following relation:

$$
S_{\mathrm{q} 0}=\varepsilon \cdot R_{\mathrm{IC}}
$$

where $\varepsilon$ is the Seebeck coefficient and is equal to $0.07 \mathrm{~V} \cdot \mathrm{K}^{-1}$ [5].

The sum $R_{\text {loss }}+R_{\text {contact }}$ can then be obtained using Eq. (A.9).

\section{A.2. Heat source in the reaction channel: calibration using the chemical reaction}

In this case, the contribution of the resistance of contact changes (see Fig. 15). The sensitivity is then defined as

$$
S_{\mathrm{q}}^{\prime \prime}=U_{\mathrm{m}}^{\prime \prime} / \dot{q}_{\mathrm{rx}}
$$

The heat released in the channel $\dot{q}_{\text {rx }}$ can be divided into two parts:

$$
\dot{q}_{\mathrm{rx}}=\dot{q}_{\mathrm{m}}^{\prime \prime}+\dot{q}^{\prime \prime} \text { loss }
$$

where $\dot{q}^{\prime \prime}$ loss is the heat flow lost equal to

$$
\dot{q}^{\prime \prime}{ }_{\text {loss }}=1 / R_{\text {loss }} \cdot \Delta T
$$

and $\dot{q}^{\prime \prime}$ is the heat flow measured by the IC membrane:

$$
\dot{q}^{\prime \prime}{ }_{\mathrm{m}}=1 /\left(R_{\text {contact }}+R_{\mathrm{IC}}\right) \cdot \Delta T
$$

From Eqs. (A.12)-(A.14), we can derive

$$
\dot{q}^{\prime \prime}{ }_{\mathrm{m}}=\dot{q}_{\mathrm{rx}} \cdot R_{\text {loss }} /\left(R_{\text {contact }}+R_{\mathrm{IC}}+R_{\text {loss }}\right)
$$

Combining Eq. (A.7) with Eq. (A.15), we get

$$
\dot{q}^{\prime \prime}{ }_{\mathrm{m}} / \dot{q}_{\mathrm{m}}^{\prime}=U_{\mathrm{m}}^{\prime \prime} / U_{\mathrm{m}}^{\prime}=\dot{q}_{\mathrm{rx}} / \dot{q} \cdot R_{\text {loss }} /\left(R_{\text {contact }}+R_{\text {loss }}\right)
$$

Comparing the sensitivity $S^{\prime \prime}{ }_{\mathrm{q}}$ obtained in this case with the one obtained in the previous paragraph $S_{\mathrm{q}}^{\prime}$, we get

$$
S^{\prime \prime}{ }_{\mathrm{q}} / S_{\mathrm{q}}^{\prime}=\left(U^{\prime \prime}{ }_{\mathrm{m}} / \dot{q}_{\mathrm{rx}}\right) /\left(U_{\mathrm{m}}^{\prime} / \dot{q}\right)=R_{\text {loss }} /\left(R_{\text {contact }}+R_{\text {loss }}\right)
$$

Knowing the sum $R_{\text {loss }}+R_{\text {contact }}$ from the first calculation and knowing $R_{\mathrm{IC}}, R_{\text {loss }}$ can be estimated and thus compared to $R_{\text {contact. }}$ 


\section{References}

[1] W. Ehrfeld, et al., Potentials and realisation of microreactors, DECHEMA Monographs, vol. 132, 1996.

[2] T.M. Floyd, et al., Novel liquid phase microreactors for safe production of hazardous speciality chemicals, in: W. Ehrfeld (Ed.), Proceedings of the Third International Conference on Microreaction Technology (IMRET 3), B.S. 2000, 2000, pp. 171- 180 .

[3] J.M. Köhler, M. Zieren, Chip reactor for microfluid calorimetry, Thermochim. Acta 310 (1-2) (1998) 25-35.

[4] J. Lerchner, et al., On-line monitoring of enzyme activities using microreactor heat power meters, in: Proceedings of the Third International Conference on Microreaction Technology (IMRET 3), 1999, pp. 469-478.

[5] J. Lerchner, A. Wolf, G. Wolf, Recent developments in integrated circuit calorimetry, J. Therm. Anal. Cal. 57 (1) (1999) 241-251.

[6] J. Lerchner, et al., Accuracy in integrated circuit (IC) calorimeters, Thermochim. Acta 382 (1-2) (2002) 65-76.

[7] S. Dushman, The rate of the reaction between iodic and hydroiodic acids, J. Phys. Chem. 8 (1904) $453-482$.

[8] M.C. Fournier, L. Falk, J. Villermaux, A new parallel competing reaction system for assessing micromixing efficiency - experimental approach, Chem. Eng. Sci. 51 (22) (1996) 5053-5064.

[9] M.C. Fournier, L. Falk, J. Villermaux, A new parallel competing reaction system for assessing micromixing efficiency — determination of micromixing time by a simple mixing model, Chem. Eng. Sci. 51 (23) (1996) 5187-5192.

[10] P. Guichardon, L. Falk, Characterisation of micromixing efficiency by the iodide-iodate reaction system. Part I. Experimental procedure, Chem. Eng. Sci. 55 (2000) 4233-4243.

[11] P. Guichardon, L. Falk, J. Villermaux, Characterisation of micromixing efficiency by the iodide-iodate reaction system. Part II. Kinetic study, Chem. Eng. Sci. 55 (2000) 4245-4253.

[12] J. Villermaux, Mixing effects on complex chemical reactions in a stirred reactor, Rev. Chem. Eng. 7 (1) (1991) 51-108.

[13] J. Villermaux, L. Falk, M.C. Fournier, Potential use of a new parallel reaction system to characterize micromixing in stirred reactors, AIChE Symp. Ser. 50 (299) (1994) 50-54.

[14] J. D’Ans, E. Lax, Taschenbuch für Chemiker und Physiker, 1949, pp. 1090-1093.

[15] M. Baerns, H. Hofmann, A. Renken, in: G.T.V.S.N. York (Ed.), Chemische Reaktionstechnik. Lehrbuch der technischen Chemie, Georg Thieme Verlag Stuttgart, New York, vol. 1, 1987, pp. 347-358.

[16] M. Baerns, H. Hofmann, A. Renken, in: G.T.V.S.N. York (Ed.), Chemische Reaktionstechnik. Lehrbuch der technischen Chemie, Georg Thieme Verlag Stuttgart, New York, vol. 1, 1987, pp. 331-336.

[17] W. Ehrfeld, et al., Characterization of mixing in micromixers by a test reaction: single mixing units and mixer arrays, Ind. Eng. Chem. Res. 38 (3) (1999) 1075-1082. 